\title{
INTELIGENCIA EMOCIONAL, MOTIVACIÓN Y RENDIMIENTO ACADÉMICO EN EDUCACIÓN INFANTIL
}

\author{
Ana Ma de Caso Fuertes \\ Universidad de León \\ amcasf@unileon.es \\ Jana Blanco Fernández \\ Universidad de León \\ Marian García Mata \\ Universidad de León \\ Alfredo Rebaque Gómez \\ Universidad de León \\ Rocío García Pascual \\ Universidad de León
}

Fecha de Recepción: 15 Febrero 2019

Fecha de Admisión: 30 Abril 2019

\section{RESUMEN}

Debido a la importancia creciente que está teniendo el estudio de la influencia de la inteligencia emocional en la educación, este trabajo pretende comprobar la influencia de un entrenamiento en emociones, relacionando este concepto con la motivación por el aprendizaje, en el rendimiento académico de los alumnos de $2^{\circ}$ curso de Educación Infantil. Para ello se utilizó una muestra de 44 participantes de un centro educativo de la ciudad de León, divididos en un grupo control y un grupo experimental al que se le aplicó el programa instruccional. Tras evaluarlos antes y después de la intervención, con un cuestionario diseñado a tal efecto, teniendo en cuenta las características psicoevolutivas de los alumnos, los resultados muestran una mejora significativa de la habilidad para la identificación de las emociones, pero no se correlaciona con un aumento en el rendimiento académico de los participantes del grupo experimental.

Palabras clave: inteligencia emocional; motivación académica; rendimiento académico; segundo ciclo de educación infantil; entrenamiento emocional

\section{ABSTRACT}

Emotional intelligence, motivation and academic performance in childhood education.

Because of the increase of the interest in the influence of emotional intelligence in education, this research aims to prove the influence of an emotional training in the academic achievement of 
preschool s children, all of it related to academic motivation. Participants were 44 children who attend to second grade of preschool. They were divided into two groups: the control one and the experimental one, which received the emotional training. Participants were assessed with a new cuestionnaire designed to this study, taking into account the children s developmental characteristics. Results show a significant improvement in the hability of emotional identification, but it doesn $t$ match with children s academic achievement ot the experimental group, as they didn $t$ get to improve their marks.

Keywords: emotional intelligence; academic motivation; academic achievement; preschoolar children; emotional training

\section{CONTEXTUALIZACIÓN}

La finalidad principal en la educación de los niños hasta hace pocos años era adquirir conocimientos básicos y adquirir las diferentes normas sociales. Esta forma de impartir conocimientos, que aún se sigue utilizando, está en proceso de modificación. Se ha demostrado que el castigo 0 hacerle sentir al niño inútil no es una buena respuesta a sus necesidades, sin embargo, la motivación es mucho más efectiva a la hora de responder a sus necesidades y también para entrenar al pequeño de forma que pueda llegar a motivarse por sí mismo, como explica Pérsico (2016).

Es por ello que, actualmente, la educación Emocional es un tema en auge. Parece claro que tanto maestros como padres (Carrillo, Estévez \& Gómez-Medina, 2018) deberían dominar estas habilidades para favorecer el desarrollo socioemocional y evolutivo de los niños, comenzando por la etapa de Educación Infantil. Educar mediante la inteligencia emocional es una labor fundamental en la educación, la cual debería hacerse mediante la práctica, con un entrenamiento de las habilidades emocionales a lo largo de la vida, para convertirlas en una respuesta a las necesidades naturales de las personas. (Fernández-Berrocal \& Ruiz, 2008)

El concepto de Educación emocional que propone Bisquerra (2013) menciona que tiene como objetivo el desarrollo de competencias emocionales, entendiendo éstas como el conjunto de conocimientos, capacidades, habilidades y actitudes necesarias para tomar conciencia, comprender, expresar y regular de forma apropiada los fenómenos emocionales. Estos fenómenos emocionales serán los que favorezcan o no una adecuada evolución del niño en adulto, de tal modo que la Educación emocional se convierte en un punto clave en la educación de los niños de Educación Infantil, ya que ésta proporcionará o no las bases adecuadas para su correcto desarrollo y aprendizaje

Según Daniel Goleman (1996), esta inteligencia está directamente relacionada con unas habilidades basadas en el reconocimiento de los sentimientos ajenos y propios sirviendo de guía para el pensamiento y la acción. Dependiendo del tipo de tareas que se realicen, se utilizarán unas u otras habilidades, ya que éstas son interdependientes. Las ventajas que tienen las personas que han adquirido estas competencias son muy positivas, ya que, aparte de estar satisfechas y cómodas consigo mismas, alcanzan el éxito y son más eficientes en su trabajo, como lo demuestran Nóbrega y Franco (2014) al concluir que existen relaciones significativas entre el número de libros leídos y la inteligencia emocional en niños de primer ciclo. También se muestran más alegres y comunican sus sentimientos con facilidad, lo que favorece la salud mental (Pérsico, 2016). Lo contrario ocurre con personas con exiguas habilidades emocionales, ya que son más propensas a sufrir estrés y diversos problemas emocionales a lo largo de la vida académica.

El propio Bisquerra (2013) ha señalado que todos los niños tienen unas necesidades sociales determinadas, las cuales deben estar reflejadas y atendidas en el currículum académico, por lo que la educación emocional sería la réplica a estas necesidades. De este modo, el propio currículo de Educación Infantil, menciona la educación emocional, de manera implícita, en los objetivos, conte- 
nidos y criterios de evaluación donde se mencionan ciertos aspectos acerca de este tema, por ejemplo, dentro del área de Conocimiento de sí mismo y autonomía personal, del segundo ciclo de educación infantil: "3. Identificar los propios sentimientos, emociones, necesidades o preferencias, y ser capaces de denominarlos, expresarlos y comunicarlos a los demás, identificando y respetando, también, los de los otros". (Real Decreto 1630/2006, de 29 de diciembre, pag., 4), por lo que parece necesario favorecer el desarrollo de competencias socioemocionales desde los primeros años de vida, proponiendo pautas de actuación como lo hacen Guil, Gil-Olarte, Serrano y Larrán (2014).

Una de las preocupaciones que nos encontramos en la actualidad y que ha causado especial interés, es determinar el papel que ejercen las emociones en la escuela y cómo afecta en el éxito académico de los estudiantes. (Jiménez \& López- Zafra, 2009). La amplitud que se tiene a la hora de atender las emociones, expresarlas con claridad y, sobre todo, recomponer ciertas actitudes negativas, en referencia al ánimo, van a afectar directamente sobre la salud y equilibrio mental, lo cual está relacionado con el rendimiento académico (Fernández- Berrocal \& Ruiz, 2008).

En primer lugar, tenemos que ser conscientes de que la inteligencia no es un factor único para determinar el éxito profesional, personal y académico de las personas, de hecho, según Pérsico (2016), para que una persona alcance el éxito necesita un 20\% de conocimientos académicos (el coeficiente intelectual) y un $80 \%$ de inteligencia emocional; por lo tanto, también influyen las emociones jugando un papel cultural en la actualidad.

La inteligencia emocional está directamente relacionada con la vida y la creatividad, sin embargo, no se puede relacionar de manera global con el rendimiento académico. (Hernández, 2005). Llegaron a esta misma conclusión Newsome, Day y Catano (2000), pero sin embargo encontraron una relación significativa acerca del rendimiento escolar mediante una prueba de habilidad cognitiva.

Si tenemos una visión racionalista extrema, se puede determinar que tanto cognición como emoción son dos variables asimétricas y opuestas (Mayer, Roberts \& Barsade, 2008), pero, sin embargo, afirmamos que permiten al sujeto un correcto funcionamiento a la hora de adaptarse al medio (Salovery \& Grewal, 2005). Tanto la regulación como la adquisición de las emociones se produce en los primeros años de vida, por lo tanto, cabe destacar, que tanto la familia como la escuela deben ser unos pilares fundamentales para potenciar el desarrollo de los más pequeños.

Un factor que, definitivamente, interfiere entre la inteligencia emocional y el rendimiento escolar es el coeficiente intelectual. Es decir, podemos encontrarnos con alumnos con un coeficiente intelectual alto pero que no son capaces de controlar sus emociones, lo cual le dificultará el éxito académico (por ejemplo, un alumno con un coeficiente intelectual alto pero que no sabe controlar su ansiedad a la hora de realizar exámenes), pero también están aquellos alumnos con un coeficiente intelectual más bajo, pero que poseen una buena inteligencia emocional, lo cual le ayudará a alcanzar el éxito académico. (Sánchez \& Hume, 2004). Sin embargo, nos encontramos con opiniones opuestas; por un lado, Barchard (2003), encuentra una relación significativa entre rendimiento académico e inteligencia emocional, relación que desaparece en cuanto se controla la variable de inteligencia académica, pero otros autores, como Bastian, Burns y Nettelbeck (2005), directamente no establecen una relación entre rendimiento académico e inteligencia emocional, aunque sí que relaciona estas variables con la satisfacción vital, situaciones estresantes o manejo de emociones. Asimismo, Belmonte, Parodi, Bermejo, Ruiz, Sainz y Belmonte-Lillo (2017), encuentran una relación positiva y significativa entre inteligencia emocional y aptitud intelectual, pero no en relación a la creatividad. Por lo tanto, podemos comprobar que el coeficiente intelectual, dependiendo de la perspectiva de la que se mire, puede influir en mayor o menor medida en las variables de inteligencia emocional y rendimiento escolar. 


\section{OBJETIVOS}

El objetivo general de este estudio es optimizar el rendimiento escolar a partir de la Inteligencia Emocional. La educación emocional está teniendo una gran importancia en la educación actual, participando en el desarrollo integral de los más pequeños, marcando un antes y un después en la visión que el hombre tiene de sí mismo y de sus capacidades. De esta forma el control emocional, el manejo de las relaciones sociales y la empatía son fundamentales en nuestra sociedad.

Para intentar alcanzar este objetivo general, se busca conocer la relación entre inteligencia emocional y rendimiento académico, además de diseñar algún instrumento que ayude a evaluar este tipo de inteligencia en los más pequeños, de tal modo que se demuestre la posibilidad de intervenir sobre el desarrollo emocional de los niños de educación infantil, mediante una instrucción adaptada a las áreas y a los contenidos temáticos de la Educación Infantil en su segundo ciclo.

\section{METODOLOGÍA}

\section{Participantes}

Han participado en este estudio 44 niños del segundo curso de Educación Infantil de un colegio concertado de la ciudad de León, de modo que se ha aplicado la intervención en una de las aulas completas de este curso: 22 niños de los cuales 11 son mujeres y 11 varones; mientras que el grupo control lo formaban los otros 22 niños de la clase homóloga del centro (12 mujeres y 10 varones). Al ser niños de segundo, su rango de edad oscila entre los 4 y los 5 años. Hay dos alumnos ACNEES, los cuales no ha sido tenidos en cuenta a la hora de realizar la propuesta de intervención, por lo que se han eliminado de la muestra, que, en caso contrario, sería de 46 niños.

El alumnado de las aulas donde se ha llevado a cabo la investigación es homogéneo, siendo el nivel socio- económico de las familias que acuden al centro medio- alto, y buscando la facilidad de la jornada partida y la posibilidad de permanecer toda la etapa escolar, desde Infantil hasta Bachillerato, en el mismo Centro Educativo.

\section{Instrumento de evaluación}

El cuestionario para evaluar el desarrollo emocional de los niños de 4 y 5 años ha sido diseñado a partir del modelo cognitivo de la Inteligencia Emocional desarrollado por los autores Mayer y Salovery, tomando como base concretamente el MSCEIT de Caruso, Mayer y Salovery (2009), el cual es un test de inteligencia emocional que pretende medir la aptitud para identificar, facilitar, comprender y manejar las emociones.

Para este estudio se ha utilizado una de las aptitudes que evalúa el MSCEIT, la percepción emocional, ya que es la aptitud más sencilla, adaptada al desarrollo de niños de 4 años. La capacidad para percibir emociones se evalúa mediante tareas de percepción de emociones en rostros faciales y fotografías, concretamente, esta adaptación está compuesta por 16 fotografías que indican una de las cuatro emociones básicas: alegría, tristeza, calma y miedo (4 ítems por cada emoción). Al lado de cada foto nos encontramos con cuatro bolas, cada una de un color, el cual representa una emoción en concreto: azul, representa la tristeza, amarillo, representa la alegría, verde, representa la calma, y negro, representa el miedo. Los niños deben rodear el color que indica qué emoción le provoca cada fotografía, de tal modo que cada ítem se evalúa del 1 al 3 (1= no logra relacionar la fotografía con la emoción que expresa; $2=$ no relaciona la fotografía con la emoción más adecuada, pero sí señala una posible emoción relacionada, en parte, con la fotografía; $3=$ ha logrado relacionar correctamente la emoción con la fotografía).

Esta prueba se pasará antes y después de la intervención sobre educación emocional que se va a impartir a los niños del grupo experimental, con la única diferencia del orden en el que se muestran las fotografías. 
Para evaluar el rendimiento académico del alumno, se han utilizado 24 ítems de los boletines de notas del segundo (antes de la intervención) y del tercer trimestre académico (después de la intervención), 8 ítems por cada una de las áreas de conocimiento de Educación Infantil: Autoconocimiento, Entorno y Lenguaje, de tal modo que se codificaron con un 1 = Necesita mejorar; 2 = Progresa adecuadamente; y 3 = Destaca.

\section{Programa de instrucción}

La intervención se llevó a cabo durante dos semanas, de modo que cada semana se entrenaba en dos emociones (alegría y tristeza la primera, y calma y miedo la segunda). Asimismo, el entrenamiento en cada emoción se producía en dos días seguidos, dejando el último día de la semana para recopilar los sentimientos y emociones vivenciadas, además de recopilar las definiciones realizadas por los alumnos de las dos emociones experimentadas.

El proceso para trabajar cada emoción era el mismo en las cuatro trabajadas, de tal modo que se iniciaba con la lectura de un cuento relacionado con la emoción correspondiente, para que los niños comprendieran los sentimientos relacionados con ella de forma lúdica. Al final de la lectura, se realizaba una serie de preguntas para comprobar si habían comprendido el significado del cuento y si alguna vez habían sentido esa emoción. Los cuentos utilizados para las diferentes emociones fueron: "Cadena de sonrisas" para la alegría; "La luna perdió su arete" para la tristeza; "La ciudad sin ruidos" para la clama; y “De qué tienes miedo?” para el miedo.

Posteriormente a la lectura del cuento, el mismo día, se les proporcionaba a los niños una ficha con una cara en blanco para hablar sobre cómo son los rasgos de la cara dependiendo de la emoción en la que nos encontremos. Con las aportaciones de todos, la maestra realizaba una cara en la pizarra, para que los niños lo dibujasen en sus hojas, comprobando así, si habían captado las ideas básicas. A continuación, entre todos, se miraban los dibujos realizados por los niños, para comprobar interpretaciones diferentes de una misma emoción.

El segundo día de trabajo con la emoción correspondiente, se dejaba a los niños realizar un dibujo libre que expresase, mediante la plástica, diferentes vivencias que les hubiesen ocurrido relacionadas con la emoción trabajada. Cada uno podía realizar el dibujo que quisiera, comprobando así en qué momentos y situaciones sienten la emoción, ya que en cada niño puede ser diferente. Además, ese día se finalizaba el entrenamiento emocional con la exposición de un cuadro de algún artista de renombre ("Los girasoles" para la alegría, "El payaso triste" para la tristeza, "El rostro" para la calma, y "El grito" para el miedo), de tal manera que se ilustraba cómo los artistas expresan muchos sentimientos mediante sus obras, y es algo que ellos mismos pueden hacer, como han demostrado anteriormente. Después de analizar el cuadro se les entregará una ficha en la que aparecía el cuadro pero sin colorear, de tal modo que los niños debían colorearlo similar al original, pero pudiendo cambiar algunos aspectos o colores, dependiendo de lo que sintiesen.

El último día de cada semana se realizaba el emocionario de las emociones trabajadas durante la misma, de tal modo que se pretendía que los niños supiesen identificar las diferentes emociones y en qué momento de sus vidas las habían sentido. Se ponían todas las aportaciones en común y quedaban registradas en una grabadora para poder analizar posteriormente las preocupaciones e inquietudes de los más pequeños. Esta actividad se realizaba al final de la semana, como recuerdo y afianzamiento del entrenamiento llevado a cabo durante la semana.

\section{Procedimiento}

Tras una exhaustiva revisión bibliográfica acerca de la necesidad de incorporar la práctica de la Inteligencia Emocional en la educación formal, se procedió a solicitar los permisos necesarios para poder llevar a cabo la investigación, de modo que no sólo se solicitó el acceso al centro a través de 
la Dirección Provincial de Educación y del equipo directivo del Centro Educativo, sino que se pidió autorización a los padres de los participantes, asegurando en todo momento la confidencialidad de los datos y el anonimato de los participantes, de tal modo que se asegura el cumplimiento de la Ley de Protección de Datos (Ley Orgánica 3/2018, de 5 de diciembre, de Protección de Datos Personales). Para solicitar todos estos permisos, previamente se había diseñado tanto el instrumento de evaluación como el programa de intervención, ya que sin la aportación de los mismos se imposibilita la aceptación de colaboración.

A partir de aquí se llevó a cabo la evaluación pretest a los 44 participantes para, a la semana siguiente, realizar la intervención específica en educación emocional a los 22 participantes que componían el grupo experimental. Mientras tanto los participantes del grupo control siguieron recibiendo los diferentes contenidos pedagógicos de acuerdo a su desarrollo, con la misma metodología tradicional que estaban recibiendo. La instrucción se llevó a cabo durante la última semana de abril y primera semana de mayo, realizada durante el horario de clase, comenzando con el cuento en la llamada "asamblea", y realizando las actividades a lo largo de la mañana. Fue la misma profesora de los niños, junto con la investigadora, la que aplicó el programa de intervención, de modo que se le daba la suficiente credibilidad y validez ante los niños y sus padres.

Una vez finalizado el periodo instruccional se aplicó el postest a los 44 participantes, de modo que se codificaron todos los datos y se llevó a cabo el análisis de los mismos mediante el programa estadístico SPSS, para poder llegar a unas conclusiones lo más ajustadas posibles.

\section{RESULTADOS}

A fin de corroborar el tipo de análisis que se debía realizar, lo primero que se analiza es la normalidad de la muestra mediante el test Kolmogorov- Smirnoff con corrección de Lilliefors para todas las variables, considerando diferencias significativas aquellas en las que el valor de $p$ es $<0,05$. En este caso, como la significación es menor a 0,05, podemos asegurar que los datos no siguen una distribución normal, por tanto, se deben realizar análisis no paramétricos.

Se comenzó el análisis no paramétrico comparando las dos muestras independientes, es decir, el grupo control, que no trabaja la educación emocional, y el grupo experimental, que trabaja la inteligencia emocional, mediante la prueba $U$ de Mann-Whitney. La tabla 1 nos muestra los resultados obtenidos.

Tabla 1.

Comparación grupo control- grupo experimental mediante la prueba $U$ de Mann- Whitney

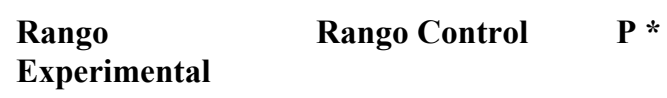

\begin{tabular}{|l|l|l|l|}
\hline $\begin{array}{l}\text { Pre Alegría Total } \\
\text { Pre Tristeza Total }\end{array}$ & 15,57 & 29,43 &, 000 \\
\hline $\begin{array}{l}\text { Pre Miedo Total } \\
\text { Pre Emoción Total }\end{array}$ & 16,98 & 28,02 &, 004 \\
\hline Post Entorno Total & 16,18 & 28,86 &, 001 \\
\cline { 2 - 3 } & 18,86 & 28,82 &, 001 \\
\cline { 2 - 3 } & *Sólo se muestran los resultados estadísticamente significativos
\end{tabular}


Si nos fijamos atentamente en la tabla 1 vemos que existen diferencias estadísticamente significativas en la identificación de las diferentes emociones, a excepción de la calma, y que estas son a favor del grupo control, como indica el mayor rango obtenido, pero estas diferencias se encuentran sólo en la evaluación pretest, lo que nos indica que los niños del grupo control identificaban mejor las emociones que los niños del grupo experimental en el inicio de la investigación.

Tras la aplicación del programa instruccional, en la evaluación postest, desaparecen esas diferencias significativas, lo que indica que los niños del grupo experimental han conseguido llegar al nivel de identificación emocional de los niños del grupo control, desapareciendo las diferencias estadísticamente significativas, motivo por el cual, estos indicadores no se reflejan en la tabla 1. Estor resultados en sí mismos ya muestran una buena eficacia del programa instruccional, al mejorar la identificación emocional de los niños del grupo experimental mientras que se mantiene estable la de los niños del grupo control.

No obstante, para avalar los datos obtenidos, se realiza también una prueba de los rangos con signo de Wilcoxon, comprobando si existen diferencias estadísticamente significativas entre la puntuación pre y la puntuación post de los niños que han recibido el programa instruccional. En este caso, nos encontramos con una significación en casi todos los valores obtenidos en la comparación de las dos aplicaciones del cuestionario, pre y post test, como indica la Tabla 2.

Tabla 2.

Comparación pre test- post test del grupo experimental mediante la prueba Wilcoxon

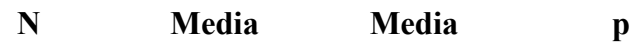

Pre test Post test

\begin{tabular}{l|l|l|l|l|}
\hline Alegría Total & 22 & 6,72 & 7,67 & 0,007 \\
$\begin{array}{l}\text { Tristeza Total } \\
\text { Calma Total }\end{array}$ & 22 & 6,56 & 7,74 & 0,001 \\
\hline $\begin{array}{l}\text { Miedo Total } \\
\text { Emoción Total }\end{array}$ & 22 & 7,00 & 8.04 & 0,001 \\
\hline \multirow{2}{*}{$\begin{array}{l}\text { Autoconocimiento Total } \\
\text { Rendimiento Total }\end{array}$} & 22 & 6,52 & 8,30 & 0,000 \\
\cline { 2 - 5 } & 22 & 26,80 & 31,47 & 0,000 \\
& 22 & 17,52 & 18,48 & 0,000 \\
\hline & *Sólo se & muestran & los resultados & estadísticamente
\end{tabular}

En este caso vemos como los alumnos han mejorado significativamente la puntuación entre la identificación de las emociones en el pretest y en el postest en todas las emociones, incluida la calma. En este caso, para una mayor comprensión, se han hallado las medias tras conocer la significación ( $p$ ), a pesar de que en las pruebas no paramétricas se trabaja con rangos.

Sin embargo, si atendemos a las variables de rendimiento académico, sólo encontramos diferencias estadísticamente significativas en una de las áreas curriculares: el autoconocimiento, además de en el rendimiento total; pero además, éstas diferencias son a favor del pretest, por lo que parece que el rendimiento baja en el tercer trimestre a pesar del entrenamiento emocional, por lo 


\section{INTELIGENCIA EMOCIONAL, MOTIVACIÓN Y RENDIMIENTO ACADÉMICO EN EDUCACIÓN INFANTIL}

que no podemos corroborar una relación entre inteligencia emocional y rendimiento académico con los datos obtenidos.

\section{DISCUSIÓN Y CONCLUSIONES}

En este trabajo nos focalizamos en un aspecto concreto del contexto escolar: el Rendimiento Académico. La revisión teórica realizada, indica que la Inteligencia Emocional está relacionada e incluso tiene una capacidad predictiva sobre el rendimiento; sin embargo, la relación existente entre ambos no es directa, ya que afectan otras variables que algunos autores han postulado, como puede ser la salud y equilibrio mental, la creatividad o el coeficiente intelectual.

Los resultados obtenidos avalan la tesis de Hernández (2005), donde afirma que la inteligencia emocional no se puede relacionar directamente con el rendimiento académico, ya que, a pesar de que los alumnos instruidos en emociones y desarrollo emocional mejoraron esta habilidad cognitiva poniéndose a la altura de los alumnos del grupo control, no lograron superar las calificaciones en rendimiento, evaluadas a través de las notas trimestrales.

No obstante, la manera de valorar el rendimiento académico de los participantes es una de las limitaciones del estudio, ya que las calificaciones se proporcionan de trimestre en trimestre y están influidas por muchos aspectos (cognitivos, comportamentales y emocionales) que pueden estar afectando a la falta de mejoría del rendimiento de los alumnos. Sería preciso pues, elaborar pruebas específicas de rendimiento académico, adaptadas al nivel de los niños, y aplicarlas antes y después del entrenamiento emocional, para confirmar la tesis mencionada anteriormente.

Además, hemos de considerar este estudio como el inicio de una línea temática, ya que existen muy pocas investigaciones que relacionen estas variables (emoción, motivación y rendimiento académico) en educación infantil, de hecho, nos hemos centrado sólo en uno de los componentes de la inteligencia emocional: la identificación de las emociones, debido a la corta edad de los participantes, pero no podemos perder de vista que la inteligencia emocional incluye otras muchas variables como son facilitación, comprensión y manejo emocional (Caruso, Mayer, Salovery, 2009).

Para concluir la importancia del presente estudio, cabe citar a Goleman (1996) cuando afirma que "ser inteligentes otorga a las emociones un papel central en el conjunto de aptitudes necesarias para vivir", es decir, hay que aprender a controlar las emociones en determinados momentos de la vida, en los que se requiere un equilibrio mental para la resolución de problemas y así poder llegar a un control de las acciones y pensamientos simultáneamente.

\section{REFERENCIAS BIBLIOGRÁFICAS}

Barchard, K. (2003) Does emotional intelligence assist in the prediction os academic succes? Educational and Psychological Measurement, 63 (5), 840- 858.

Bastian, V.A., Burns, N.R., \& Nettelbeck, T. (2005). Emotional Intelligence predicts life skills, but not as well as personality and cognitive abilities. Personality and Individual Differences, 39 (6), 1135-1145.

Belmonte, V., Parodi, A., Bermejo, R., Ruiz, M.J., Sainz, M. \& Belmonte-Lillo, V. (2017). Relaciones entre aptitud intelectual, inteligencia emocional y creatividad en alumnado de ESO. International Journal of Developmental and Educational Psychology, 1 (1), 35-44.

Bisquerra, R. (2013). Educación Emocional. Propuestas para educadores y familias. Bilbao: DESCLÉE DE BROUWE.

Carrillo, A., Estévez, C., Gómez-Medina, M.D. (2018). ¿Influyen las prácticas educativas en el desarrollo de la Inteligencia Emocional de sus hijos? International Journal of Developmental and Educational Psychology, 1 (1), 203-212.

Caruso, D.R., Mayer, J.D., \& Salovery, P. (2009). Test de inteligencia emocional. Madrid: TEA 
Ediciones.

Fernández- Berrocal, P. \& Ruiz, D. (2008). La inteligencia emocional en la Educación. Revista Electrónica de Investigación Psicoeducativa, 6 (2), 421-436.

Goleman, D. (1996). Inteligencia emocional. Barcelona, España: Kairós.

Guil, R., Gil-Olarte, P., Serrano, N. \& Larrán, C. (2014). Desarrollo de la Inteligencia Emocional de 0 a 3 años. International Journal of Developmental and Educational Psychology, 1 (2), 585-594.

Hernández, P. (2005). ¿Puede la Inteligencia Emocional predecir el rendimiento? Potencial predictor de los Moldes Mentales. Revista Interuniversitaria de Formación del Profesorado, 19 (3), 45-62.

Jiménez, M.I., \& López- Zafra, E. (2009). Inteligencia emocional y rendimiento escolar: estado actual de la cuestión. Revista Latinoamericana de Psicología, 41 (1), 69-79.

Mayer, J.D., Roberts, R.D. \& Barsade, S.G. (2008). Human Abilities Emotional Intelligence. The Anuual Review of Psychology, 59, 507-536.

Newsome, S. Day, A.L. \& Catano, V.M. (2000). Assessing the predictive validity of emotional intelligence. Personality and Individual Differences, 29, 1005-1016.

Nóbrega, N. \& Franco, G. (2014). Inteligencia emocional y actividad lectora en una escuela de 1ำ ciclo. International Journal of Developmental and Educational Psychology, 5 (1), 159-166.

Pérsico, L. (2016). Inteligencia emocional. Madrid, España: LIBSA.

Real Decreto 1630/2006, de 29 de diciembre, por el que se establecen las enseñanzas mínimas del segundo ciclo de Educación Infantil. (BOE núm. 4, 3 de mayo de 2006).

Salovery, P. \& Grewal, D. (2005). The Science of Emotional Intelligence. Current Directions in Psychological Science, 14, 281-285

Sánchez, M.T. \& Hume, M. (2004). Evaluación e intervención en inteligencia emocional y su importancia en el ámbito educativo. Revista de Docencia e investigación, 14, 1-26. 
